\title{
Space storms as natural hazards
}

\author{
L. I. Dorman ${ }^{1}$, N. G. Ptitsyna ${ }^{2}$, G. Villoresi ${ }^{3}$, V. V. Kasinsky ${ }^{4}$, N. N. Lyakhov ${ }^{4}$, and M. I. Tyasto ${ }^{2}$ \\ ${ }^{1}$ Israel Cosmic Ray Center affiliated to Tel Aviv University and Technion, Qazrin, Israel \\ ${ }^{2}$ SPbIZMIRAN, St. Petersburg, Russia \\ ${ }^{3}$ University "Roma Tre", Rome, Italy \\ ${ }^{4}$ Irkutsk Institute of Railway Transport, Irkutsk, Russia
}

Received: 18 June 2007 - Revised: 6 December 2007 - Accepted: 19 December 2007 - Published: 10 April 2008

\begin{abstract}
Eruptive activity of the Sun produces a chain of extreme geophysical events: high-speed solar wind, magnetic field disturbances in the interplanetary space and in the geomagnetic field and also intense fluxes of energetic particles. Space storms can potentially destroy spacecrafts, adversely affect astronauts and airline crew and human health on the Earth, lead to pipeline breaking, melt electricity transformers, and discontinue transmission. In this paper we deal with two consequences of space storms: (i) rise in failures in the operation of railway devices and (ii) rise in myocardial infarction and stroke incidences.
\end{abstract}

\section{Introduction}

Electromagnetic fields and plasma processes originating in the Sun produce space storms and related geomagnetic disturbances which could adversely affect technological systems functioning in space and on the Earth and can be potentially dangerous for human health (see e.g., Allen and Wilkinson, 1993; Roederer, 1995; Ptitsyna et al., 1998; Jansen et al., 2000; Cherry, 2002; Iucci et al., 2005, and references therein). Geomagnetic storms produce induced currents (GIC) in ground-based long conductors with low resistivity: power lines, telecoms cables, pipelines, and railway lines. Intense GIC could lead to failures in functioning of above technological systems. (Lanzerotti, 1979; Boteler et al., 1998; Pirjola et al., 2005; Ptitsyna et al., 2005). In this paper, we present results on hazard to technology, specifically, to railway automatic control systems, signaling and switching devices (hereafter named "railway devices"). In addition, to widen the knowledge on the implications of space storm hazards, we present some results on adverse effects of space storms to human health.

Correspondence to: L. I. Dorman

(lid@physics.technion.ac.il)

\section{Hazard to railway devices}

GIC can hamper rail traffic by disturbing railway devices. Such a case was reported by US newspapers in relation to the historical geomagnetic storm of 13-15 May, 1921 (Newspapers Archive): "At 7:04 AM on 15 May, the entire signal and switching system of the New York Central Railroad below 125th street was put out of operation, followed by a fire in the control tower at 57th Street and Park Avenue. The cause of the outage was later ascribed to a 'ground current' that had invaded the electrical system. Railroad officials formally assigned blame for a fire destroyed the Central New England Railroad station, to the aurora. Over seas, in Sweden a telephone station was 'burned out', and the storm interfered with telephone, telegraph and cable traffic over most of Europe".

To study links between failures in railway devices (anomalies) and space storms we analyzed the anomalies together with geomagnetic indices $D_{s t}$ and $A p$ (or the local index $A$ ), which describe the level of geomagnetic disturbances. The anomalies were registered daily in 2004 on the East Siberian Railway (geomagnetic latitude $\phi_{m}=46^{\circ} \mathrm{N}-51^{\circ} \mathrm{N}$; geomagnetic longitude $\lambda_{m}=168^{\circ} \mathrm{E}-187^{\circ} \mathrm{E}$ ). The anomalies consist mainly in unstable functioning and failures in automatic block-system (track circuit, switches, control devices, etc.) often resulting in false engagement of railway tracks and a red signal instead of a green one. Data were cleaned up by anomalies due to meteorologically connected reasons, mechanical wire breakage, staff errors, vandalism, etc. The remaining 2176 anomalies have been used in the analysis.

\subsection{Results}

The overall correlation between the anomalies and geomagnetic indices Ap and Dst was found to be extremely small $(\sim 0.1)$. This result is not surprising since initial data cleaned up by non-geomagnetic causes could still include breakdowns caused by different technical factors independent on

Published by Copernicus Publications on behalf of the European Geosciences Union. 


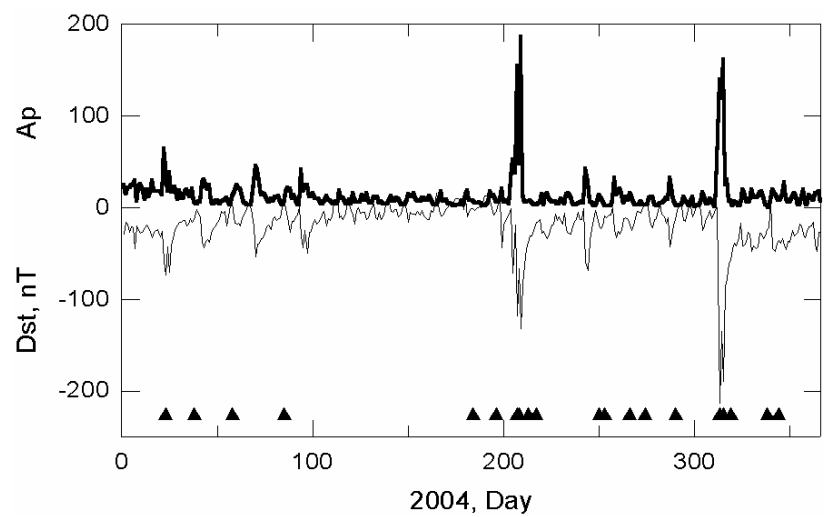

Fig. 1. Distribution of anomalies in railway automatic blocksystems not related to recognized technical malfunctions (black triangles). Geomagnetic activity: $D_{s t}-$ black line, $A p-$ grey line.

geomagnetic effects. Moreover, GIC induced in railway automatic control systems, signaling and switching devices should probably exceed a certain threshold to cause a failure or breakdown. Therefore, to reveal possible relationships between anomalies and geomagnetic activity, we concentrated on the events for which this relation is most probable, namely, on the disturbed periods of geomagnetic activity, when induced currents are maximal, and, on the other hand, on the breakdowns for which known technical causes were not revealed.

For the last case, we chose the failures for which it was reported in the original reports: "all devices are in normal state, no reasons for their anomalous functioning have been found". There were 20 of such cases in 2004. Figure 1 presents the time distribution of these anomalies together with $A p$ and $D_{s t}$ profiles. There was very low geomagnetic activity during three months, 1 April-30 June (mean $A p=9$, $D_{s t}=-6$ ). The anomaly distribution shows a similar pattern: no anomalies occurred in this "quiet" period, and the majority of the anomalies shows a tendency to concentrate around disturbed geomagnetic periods. The probability of anomaly occurrence is 20/365, which gives about 5\%. In 2004, during geomagnetic storms there were 19 disturbed days in which $D_{s t}<-50$; six anomalies occurred during these days, which gives a probability of anomaly occurrence of about $30 \%$. Therefore, the probability of anomaly occurrence in geomagnetically disturbed periods is 6 times higher than the average anomaly occurrence.

It is of interest to notice that the anomalies are distributed all along the storm evolution, in the main phase, as well as in the recovery phase. This feature seems to suggest that GIC drivers and their effectiveness in producing anomalies might be different even during a single storm, depending on the storm phase. This is in agreement with Iucci et al. (2005), in which it was found that the occurrence of satellite anomalies depends on the storm phase and satellite orbit.
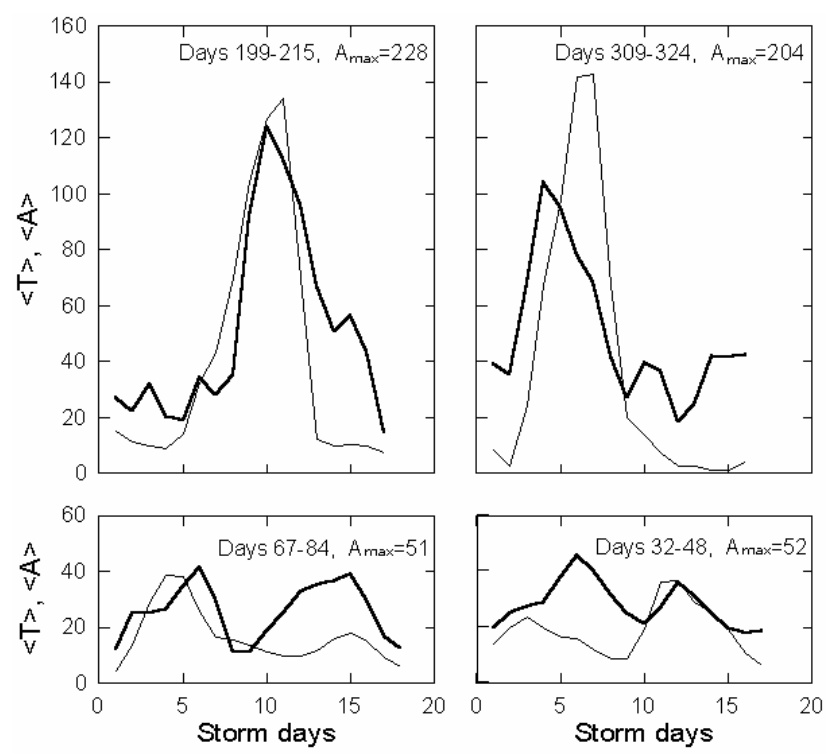

Fig. 2. Daily sum of duration $T$ (in minutes) of anomalies to signaling and train control equipment vs. days from the geomagnetic storm onset. Black line: the moving 5-day average of anomaly duration $<T>$ (in $\mathrm{min} / 10$ ); Grey line: the moving 5-day average of local geomagnetic index $<A>$.

To study the possible links between geomagnetic storms and severity of the anomalies (proportional to duration $T$ of the anomaly) we selected major and severe geomagnetic storms characterized by local index $A_{\max }>50$, and computed for each storm: (i) the daily sum of $T$ (in minutes), (ii) the moving 5-day average of anomaly durations, $\langle T\rangle$, and of local geomagnetic index, $\langle A>$, and (iii) the correlation between $<T>$ and $<A>$. In Fig. 2 we present examples of $<T>$ distribution during the following periods of two superstorms $\left(A_{\max } \sim 200\right.$ ) accompanied by multiple storm sudden commencements (SSC): 17 July-2 August (days 199-215) and 4-19 November (days 309-324) and two storms with $A_{\max } \sim 50$, not accompanied by SSC: 1-17 February (days 32-48), 7-24 March (days 67-84). It is seen that the intensity of anomalies (characterized by the duration of anomalies $<T>$ ) is in most cases geomagnetically dependent, though the level of correlation between duration of anomalies and geomagnetic disturbances can be different. The panels show examples of high (two upper panels) and low (two lower panels) correlation. A significant increase of anomaly durations (by a factor of $\sim 3$ ) during storms is observed. A high degree of correlation is found for periods of two superstorms in 2004: for 5-22 November $\left(A_{\max }=204\right.$, correlation coefficient $K=0.71)$ and 17 July-2 August $\left(A_{\max }=228, K=0.83\right.$ ). The lower left panel seem to show that, as the local geomagnetic activity declines in February, the duration of anomalies increases for half the period shown and the two parameters move together for the second half. The panel on the lower right shows about equal correspondence for the first part of 


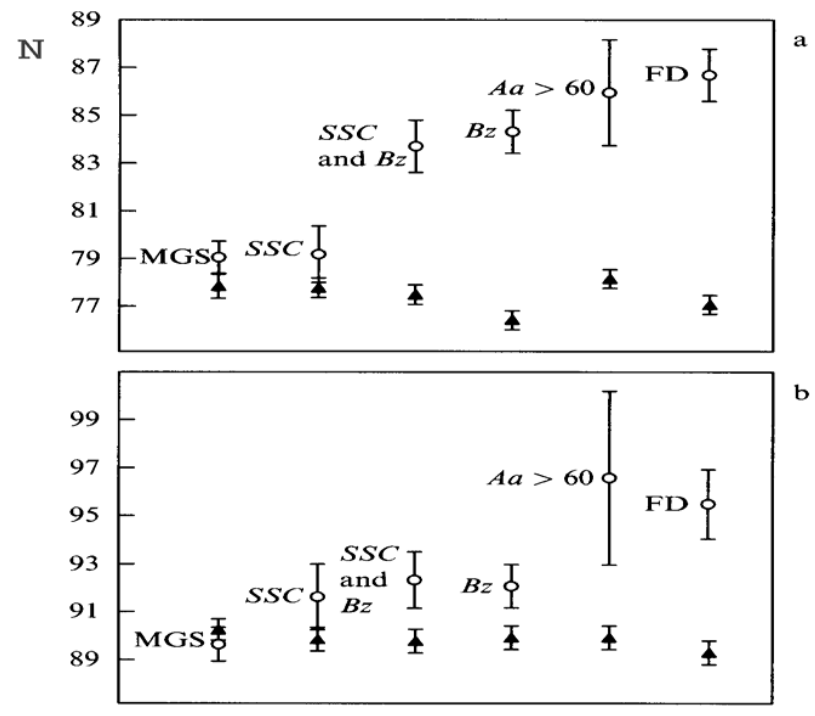

Fig. 3. Daily mean incidence rate $N$ for myocardial infarction and brain stroke during space storms defined by the following parameters: MGS, major geomagnetic storms; SSC sudden commencement (arrival to the Earth the interplanetary shock wave); $B z$, existence of sharp increase and rotation of interplanetary magnetic field $B$ to vertical negative direction; $A a$, geomagnetic activity index; FD, decreasing phase of Forbush effect in cosmic ray intensity. The filled triangles indicate the mean values of infarctions and brain strokes during quiet days (not included in the corresponding criterion of selection).

the period in March and an increase in anomaly duration during the second part without corresponding increased longlasting local geomagnetic disturbance. The correlation coefficients for the periods of 1-17 February $\left(A_{\max }=52\right)$ and 7-24 March $\left(A_{\max }=51\right)$, were $K=0.17$ and $K=0.43$, respectively. This result might support the idea that the onset of extended geomagnetic activity triggers longer duration anomalies.

\section{Hazard to human health}

In the last decades many investigations have been carried out on the influence of space storms through the action of natural geomagnetic field, upon the morbidity in different diseases (e.g. Roederer, 1995; Ptitsyna et al., 1998; Cherry, 2002, and references therein). Though magnitudes involved are very small in comparison to local intracellular electric and magnetic fields, emerging bodies of empirical findings and laboratory results support the possibility that the association has a real biophysical basis. We present results of studies on pathology rates in Russia in relation to space storms (see e.g. Villoresi et al., 1994, 1995, 1998).

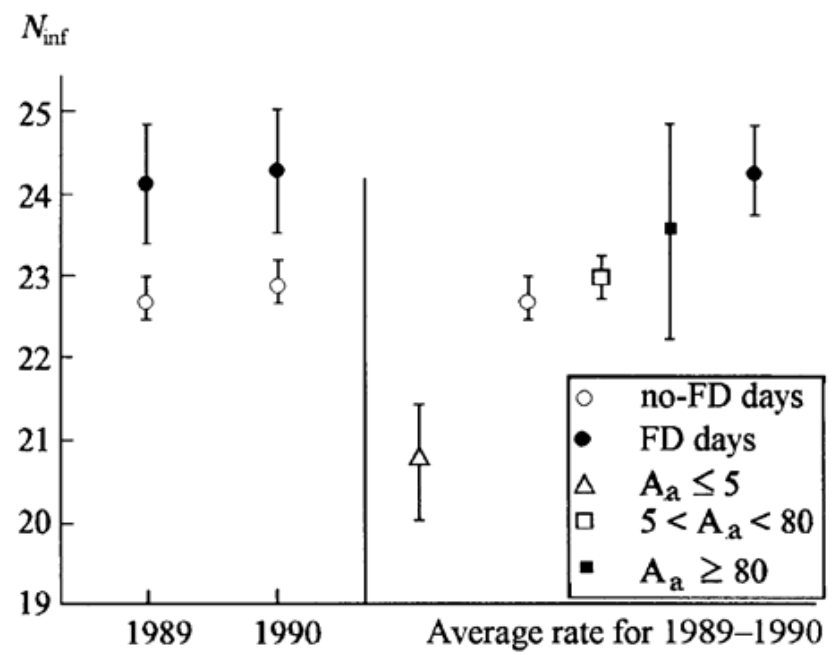

Fig. 4. The daily mean infarction rate for period 1989-1990 and separately for 1989 and 1990 for days chosen by the following criteria: (black circles) FD days, geomagnetically perturbed days corresponding to the decreasing phase of Forbush effects in cosmic ray intensity; (open circle) no-FD days, all days except FD days defined above; (open triangle, open square, open rombus) for days with $A a \leq 5 ; 5<A a<80 ; A a \geq 80$, respectively.

\subsection{Data}

Medical parameters. The numbers of daily cases of selected diseases registered by Medical Emergency Services in St. Petersburg and in Moscow have been analyzed. The data were: (i) St. Petersburg 1981: Total calls (1 314200 cases), Infarctions (14 248), Psychiatric (14 067); (ii) Moscow 1979-1981: Total calls (6304 000 cases), Myocardial Infarction (85 819), Brain Stroke (98625), Hypertension (165 699), Sudden Death (71753), Heart Rhythm Disturbance (146545), Epilepsy (53613).

Additionally, the numbers of daily admissions for infarction (15 543 cases) in the 14 greatest hospitals in St. Petersburg during 1989-1990 have been analyzed.

Space storms parameters. To characterize space storms and related geomagnetic storms we selected major geomagnetic storms (MGS) according to NOAA classification $(100>A p>49)$, the geomagnetic activity index $A a$, and some interplanetary parameters: existence of SSC (storm sudden commencement), sharp increase in the negative $B z$ component of the interplanetary magnetic field and decrease in cosmic ray intensity (Forbush decrease, FD). The days of FD descending phase, i.e., from the beginning of the decrease to the minimum of intensity level were chosen as magnetically disturbed days. 


\section{Results}

The medical data cleaned up by meteorological (annual) and social effects have been analyzed in connection with enhanced levels of geomagnetic activity as defined by the previously reported parameters. It was found that only myocardial infarction and brain stroke were sensitive to space storms. In Fig. 3 we show the obtained results for Moscow. Different characterization of geomagnetic activity levels usually correspond to different levels in pathology rates. The most remarkable and statistically significant effect has been observed during days of space storms coinciding with the days of the declining phase of cosmic-ray FD. During these days the average numbers of infarctions and strokes registered by Emergency Services increase by a factor $1.125 \pm 0.015$, $1.070 \pm 0.017$ respectively, i.e. increase by $\sim 12$ and $7 \%$ respectively. Anyway, there are interesting implications in the other space weather comparisons to numbers of infarction calls. For example, when there is both a storm sudden commencement (SSC) and negative interplanetary magnetic field at geostationary altitude $(B z)$ then the number of infarctions appears to increase possibly significantly over the number on quiet days. However, if only $\mathrm{Bz}$ is considered, the number of increased infarctions is greater although possibly the difference is statistically insignificant.

In Fig. 4 we show results for St. Petersburg 1989-1990. For infarction data, different levels of geomagnetic activity, defined by the Aa index, seem to be correlated with the myocardial infarction rate in a regular way: the higher infarction incidence being observed in days of higher magnetic activity, albeit with low statistical significance. The best indicator of geomagnetically perturbed days is the cosmic-ray FD effect: there is a statistically significant increase, $6.5 \pm 2.0 \%$, in the infarction rate during FD days relative to all other days (noFD days).

For St. Petersburg 1981 infarction data, we found that during FD-days the average rate of myocardial infarction increases by a factor $1.14 \pm 0.06$.

The average value of the obtained results for Moscow 1979-1981, St. Petersburg 1981, and 1989-1990 gives an increase in myocardial infarction incidence rate during FD days of $10.5 \pm 1.2 \%$.

\section{Conclusions}

We presented in this work results based on statistical data for links between geomagnetic storms and anomalies in the operation of railway automatics and telemetry. A significant increase of the anomaly duration (by $\sim 3$ times) during storms is observed. The probability for the anomalies not related to recognized technical malfunctions to occur in disturbed periods is 6 times higher than for the average anomaly occurrence.
We also gave evidence of harmful potential of space storms for human health: infarction and brain stroke increase during space storms, especially in days corresponding to the descending phase of cosmic ray Forbush decrease events (by 10 and $7 \%$ respectively).

However, our statistical results should be verified by using larger datasets and additional methods of analysis. This will be the aim of subsequent studies.

Acknowledgements. We would like to thank the Block-System Department of East Siberian Railway for data on failures. We also are grateful to reviewers for their assistance in evaluating this paper and useful comments.

Edited by: P. Lagos

Reviewed by: J. Allen and P. Velinov

\section{References}

Allen, J. H. and Wilkinson, D. C.: Solar-terrestrial activity affecting systems in space and on Earth, in Proceedings of the Workshop Solar-Terrestrial Predictions-IV (Ottawa, Canada, May 18-22, 1992), 1993

Boteler, D., Pirjola, R., and Nevanlinna, H.: The effects of geomagnetic disturbances on electrical systems at the Earth surface, Adv Space Res., 22, 1, 17-27, 1998.

Cherry, N.: Shumann resonances, a plausible biophysical mechanism for human health effects of solar/geomagnetic activity, Nat Hazards 26, 279-331, 2002.

Iucci, N., Levitin, A. E., Belov, A. V., Eroshenko, E. A., Ptitsyna, N. G., Villoresi, G., Chizhenkov, G., Dorman, L. Parisi, M., Tyasto, M.: Space weather conditions and spacecraft anomalies in different orbits, Space Weather, 3, S01001, doi:10.1029/2003SW000056, 2005.

Jansen, F., Pirjola, R., and Favre, R.: Space Weather, Hazard to the Earth?, Swiss Reinsurance Company, Zurich, 2000.

Lanzerotti, L.: Geomagnetic influences on man-made systems, J. Atmos. Terr. Phys., 41, 787-796, 1979.

Newspaper Archive: Sunspot credited with rail tie-up, New York Times, May 16, 1921.

Pirjola, R., Kauristie, K., Lappalainen, H., Viljanen, A., and Pulkkinen, A.: Space weather risk, Space Weather, 3, S02A02, doi:10.1029/2004SW000112, 2005.

Ptitsyna, N., Villoresi, G., Dorman, L. I., Iucci, N., and Tyasto, M. I.: Natural and man-made low-frequency magnetic fields as a potential health hazard, Physics-Uspechy (Advances in Physical Sciences) 41, 7, 687-709, 1998

Ptitsyna, N. G., Kasinskii, V. V., Lyahov, N. N., Dorman, L. I., Villoresi, G., and Iucci, N.: Relationship between geomagnetic disturbances and failures in railway automatic signaling and train control equipment, Abstract, 2nd European Space Weather Week, Noordwijk, The Netherlands, Nov 2005.

Roederer, J. G.: Are magnetic storms hazardous to your health? EOS, Transactions, American Geophysical Union 76, 441, 444 445, 1995.

Villoresi, G, Kopytenko, Y.A., Ptitsyna, N. G., Tyasto, M. I., Kopytenko, E. A., Iucci, N., and Voronov, P. M: The influence of geomagnetic storms and man-made magnetic field disturbances on 
the incidence of myocardial infarction, Phys. Med., 10, 107-117, 1994.

Villoresi, G. Breus, T. K., Dorman, L. I., Iucci, N., and Rapoport, S. I.: Effect of interplanetary and geomagnetic disturbances on the rise in the number of clinically severe pathologies (myocardial infarction and stroke), Biophysics, 40(5), 983-993, 1995.
Villoresi, G., Ptitsyna, N. G., Iucci, N., and Tyasto, M. I.: Myocardial infarction and geomagnetic disturbances: analysis of morbidity and mortality data, Biophysics, 43(4), 587-595, 1998. 